(2014). Temporal Learning Analytics for Adaptive Assessment. Journal of Learning Analytics, 1 (3), 165-168.

\title{
Temporal Learning Analytics for Adaptive Assessment
}

\author{
Zacharoula Papamitsiou and Anastasios A. Economides \\ University of Macedonia, Greece \\ papamits@uom.edu.gr and economid@uom.gr
}

\begin{abstract}
Accurate and early predictions of student performance could significantly affect interventions during teaching and assessment, which gradually could lead to improved learning outcomes. In our research, we seek to identify and formalize temporal parameters as predictors of performance ("temporal learning analytics" or TLA) and examine students' temporal behaviour during testing (i.e., in terms of time-spent). The goal is to specify a functional set of parameters that will be embedded in an adaptive assessment system in order to contribute towards the personalization of feedback services. In this paper, we present the motivation and rationale for our work, along with our methodology, initial results, contributions so far, and plans for future work.
\end{abstract}

KEYWORDS: Temporal learning analytics, adaptive assessment, prediction of performance

\section{INTRODUCTION}

Researchers attempt to identify and understand the most significant factors (i.e., with the highest impact or predictive capabilities) that affect student performance in order to use them in developing automated, data-driven, and adaptive assessment systems that support students to improve their performance. It is important to build systems that will allow students to monitor their own progress, support self-regulated learning, and help them evaluate and adjust their learning strategies to increase goal achievement.

In our research, we have focused on the determination of predictive factors of student performance and on the discovery of causes of positive learning outcomes. More specifically, we explore whether associating students' self-perceptions (e.g., goal expectancy) with temporal trace data (e.g., total time to answer correctly) and coding their participation and engagement in terms of "time-spent" (e.g., uncertainty) could lead to a complementary dimension of a more concise predictive model. The research goal is to specify a functional set of parameters (latent, composite, or simple) that will be embedded in an adaptive assessment system that will provide personalized feedback services.

\section{LITERATURE REVIEW}

The most common indicators of performance explored by previous researchers include grades (in prerequisite courses, during assessment quizzes, and in their final scores) and student participation, enrollment, and engagement in activity (e.g., login frequencies, number of chat/discussion messages, etc.) (Macfadyen \& Dawson, 2010; Romero-Zaldivar, Pardo, Burgos, \& Kloos, 2012). Researchers have also examined the effect of student response time on the prediction of their performance, modelled 
(2014). Temporal Learning Analytics for Adaptive Assessment. Journal of Learning Analytics, 1 (3), $165-168$

engagement in terms of time that students are willing to spend during a course, and the relationships between study-time and motivation (Nonis \& Hudson, 2006; Xiong, Pardos \& Heffernan, 2011) with encouraging results.

\section{METHOD}

For the needs of our research, we configured a simplified version of the LAERS assessment environment (Papamitsiou, Terzis, \& Economides, 2014). We implemented a testing mechanism and a tracker that logs student temporal activity data during testing. The collected dataset includes the following (for each student): student ID, the question the student works on, the answer the student submits, the correctness of the submitted answer, how many times the student views each question, how many times the student changes the answer, the timestamp the student starts viewing a question, the timestamp the student chooses to leave the question (saves an answer), the idle time the student spends viewing each question (not saving an answer, but choosing to see another question). We also embedded into the system a pre-test questionnaire in order to measure each student's goal expectancy (GE) (a measure of student goal orientation and perception of preparation; Terzis \& Economides, 2011).

Two case studies have been conducted so far: in the first, 96 Greek high school student participants (54 females [56.3\%] and 42 males [43.7\%], aged 16 years old) attended a midterm computer-based testing exam. The 12 multiple choice questions of the test were related to the basic concepts of Informatics and Computer Programming. The testing procedure replaced the traditional paper midterm and was obligatory for all students. In the second case study, 259 Greek undergraduate university students (148 females [56.9\%] and 111 males [43.1\%], aged 18-25 years old), participated in a computer-based testing and a computerized-adaptive testing procedure for the Computers II course (related to databases, information systems, and introduction to e-commerce). The participation in the midterm exams procedure was optional. In both studies, as external motivation to increase their effort, their score would count for $30 \%$ of their final grade.

We adopted the partial-least-squares (PLS) analysis method (Chin, 1998) for the construction of a causal dependencies path diagram containing the structural and measurement model for the statistical testing of research hypotheses.

\section{RESULTS}

The following results are from the first case study only. The results for the research hypotheses are illustrated in Figure 1. The model explains the almost $63 \%$ variance $\left(R^{2}\right)$ in Actual Performance (AP). In our model, Total Time to Answer Correct (TTAC) and Total Time to Answer Wrong (TTAW) are the temporal factors that have a statistically significant effect on AP. 
(2014). Temporal Learning Analytics for Adaptive Assessment. Journal of Learning Analytics, 1 (3), 165-168.

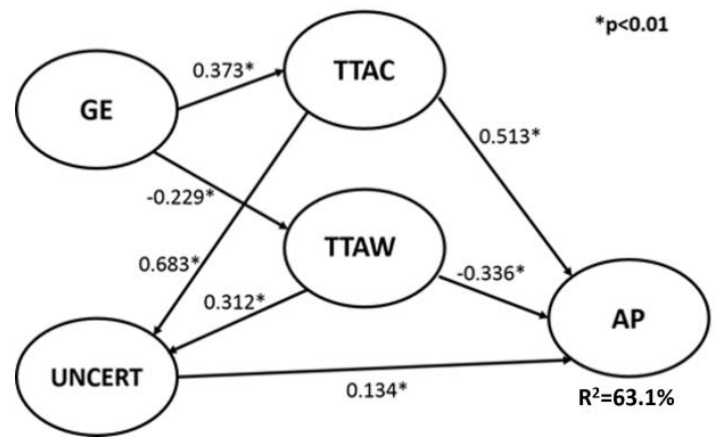

Figure 1: Path coefficients of the research model, first case study

\section{CONTRIBUTION AND WORK IN PROGRESS}

In our research, we configured the LAERS assessment environment in order to track student temporal activity data and compute the corresponding variables. We also embedded a questionnaire into the system in order to gather self-reported data. Initial results (Papamitsiou, Terzis, \& Economides, 2014) highlighted a detected trend that TTAC and TTAW have a significant direct positive and negative effect on AP respectively, and they both have a significant direct positive effect on (un-)certainty. Moreover, GE is a determinant of TTAC and TTAW, and (un-)certainty is a determinant of AP as well. In a sense, (un)certainty seems to increase student effort to answer the questions. In addition, the detected indirect effect of GE on (un-)certainty indicates that student perception of preparation affects their cautiousness (Papamitsiou \& Economides, 2014).

Our goal was to explore the logged temporal trace data and identify their possible effects on student performance and on their predictive capabilities. To this point, the contribution of this paper is that the tools and methodology adopted so far have revealed the existence and the relationships of numerous temporal parameters. For example, Total Idle Time (TIT) is an indicator of (un-)certainty, while TTAC and TTAW imply the difficulty of the given task. As a next step, it would be interesting to apply process mining in order to investigate the nature of the temporal dimension of learning more deeply. We also aim to associate TLA to Weiner's attribution theory on causes of motivation and achievement (Weiner, 1985). Further, we intend to examine emotional data collected during testing in order to discover possible relations to the existing temporal model for predicting actual performance.

Currently, we collect temporal data (e.g., TTAC, TTAW, TIT, etc.), analyze them, and explore their capabilities in order to figure out whether such user-generated trace data might constitute an appropriate, cost-effective solution towards the personalization of computerized adaptive testing services. As a future direction, we are planning to embed temporal parameters into the LAERS system to guide recommendations regarding the next most appropriate testing item, and evaluate the performance and accuracy of this type of recommender system. 


\section{REFERENCES}

Chin, W. W. (1998). The PLS approach to structural equation modeling. In G. A. Marcoulides (Ed.), Modern business research methods (pp. 295-336). Mahwah, NJ: Lawrence Erlbaum Associates.

Macfadyen, L. P., \& Dawson, S. (2010). Mining LMS data to develop an "early warning system" for educators: A proof of concept. Computers \& Education, 54(2), 588-599.

Nonis, S. A., \& Hudson, G. I. (2006). Academic performance of college students: Influence of time spent studying and working. Journal of Education for Business, 81(3), 151-159.

Papamitsiou, Z., \& Economides, A. A. (2014). Students' perception of performance vs. actual performance during computer-based testing: A temporal approach. Proceedings of the 8th International Technology, Education and Development Conference (INTED '14), Valencia, 401411.

Papamitsiou, Z., Terzis, V., \& Economides, A. A. (2014). Temporal learning analytics during computer based testing. Proceedings of the 4th International Conference on Learning Analytics and Knowledge, 31-35. New York: ACM.

Romero-Zaldivar, V-A., Pardo, A., Burgos, D., \& Kloos, C. D. (2012). Monitoring student progress using virtual appliances: A case study. Computers \& Education, 58(4), 1058-1067.

Terzis, V., \& Economides, A. A. (2011). The acceptance and use of computer based assessment. Computers \& Education, 56(4), 1032-1044.

Weiner, B. (1985). An attributional theory of achievement motivation and emotion. Psychological Review, 97, 548-573.

Xiong, X., Pardos, Z., \& Heffernan, N. (2011). An analysis of response time data for improving student performance prediction. Proceedings of the workshop on Educational Data at the $17^{\text {th }}$ Conference on Knowledge Discovery and Data Mining (ACM SIGKDD), 103-108 